\title{
Die Slowakei im Übergang
}

\section{Erhard Taverna}

erhard.taverna[at]saez.ch

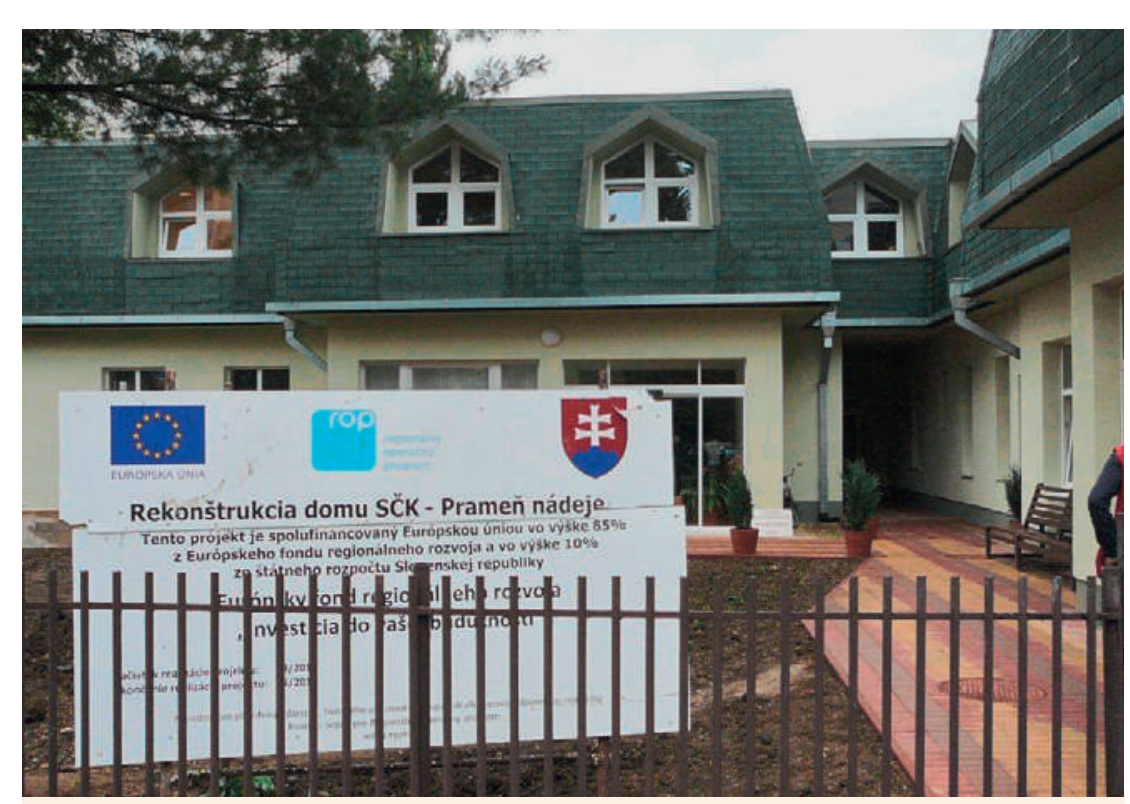

Mit EU-Beiträgen erbautes Pflegeheim in Trebisov.

Die energische Dame vom Roten Kreuz führt uns durch die zukünftige Pflegeabteilung. Noch wird verputzt und werden Kabel verlegt, Zementstaub füllt den Korridor. Das heruntergekommene Personalhaus steht am Rande des Spitalgeländes von Trebisov. Leerstehende Gebäude suchen weitere Mieter, die Bettenzahl der wenig mehr als 40 Jahre alten Klinik wurde halbiert. Das Einzugsgebiet ist geschrumpft, weil die Bezirksgrenzen nach der Wende neu gezogen wurden. 400 Betten sind immer noch zu viel, doch ausserhalb gibt es wenig. Fabriken wurden geschlossen, viele junge Menschen sind auf Arbeitssuche weggezogen.

Das Land, etwas grösser als die Schweiz, ist im Osten ärmer als im Westen. Wer früher den Sozialismus aufgebaut hat, ist jetzt alt geworden, versteht oft die Welt nicht mehr und ist für jede Hilfe dankbar. Beratung und ambulante Dienste sind im Aufbau, neuwertiges Gebrauchsmaterial aus der Schweiz hochwillkommen. Der überdimensionierte Plattenbau ist dabei zu zerfallen, viele Balkone hängen schief, weil die Armierung durchrostet. Auf dem weiten Vorplatz wuchert Unkraut, wo einst Springbrunnen plätscherten, liegt Abfall. Für den Abbruch fehlt das Geld, man improvisiert.

Das regionale Rote Kreuz hat aus eigener Initiative schon viel erreicht. Der eine Flügel des gepachteten Kinderheims erwartet die ersten Rentner, im anderen Teil wohnen einige Frauen mit Kindern. Sie sind von zu Hause geflüchtet und belegen hier Kajütenbetten, bis das Sozialamt eine neue Wohnung ge-

$\longrightarrow$ funden hat. Webstühle und Unterrichtsräume erleichtern den Aufenthalt. Drei- bis Vierbettzimmer sind auch in dem mit EU-Fördergeldern neu gebauten Pflegeheim üblich, für Privates bleibt wenig Raum. Im zweigeschossigen Haus gibt es keinen Bettenlift, und das Plastikgeschirr wird von Hand gewaschen. Wieder einmal ist es heilsam, diese Realität an unseren überbordenden Ansprüchen zu messen.

Pionierarbeit kann auch die Stadt Hummenné vorweisen. Neben ausschliesslich staatlich finanzierten gibt es auch private Unternehmungen. Der neu erbaute Pavillon entstand auf der grünen Wiese am Rande der Stadt. Wer hier liegt, muss, zusätzlich zur Grundrente, einen etwa gleich hohen, privaten Beitrag aufbringen. Die Vierbettzimmer sind videoüberwacht, die Luft wird gefiltert. Es gibt Spielgruppen, und in der gemütlichen Cafeteria, mit Blick auf den Garten, plaudern Angehörige mit Pensionären. Wie in allen besuchten Spitälern und Heimen, ist auch hier ein Teil der seit 2010 mit 91 Sattelschleppern aus der Schweiz gelieferten 1829 Betten und weiteren Geräts in Gebrauch.

Unterwegs zu weiteren Orten wie Kosice, Presov, Vranov und Medzilaborce bleibt viel Zeit, um mit Spitaldirektoren, Pflegedienstleiterin, Pressesprecherin oder Dolmetscherin zu diskutieren. Rings um die schön renovierten Stadtzentren wuchern Reklametafeln und hässliche Flachbauten in die weite, hügelige Landschaft, wo ausgedehnte Wälder, weite Wiesen und Äcker vorbeiziehen. Selten sind Kühe zu sehen, denn auch die Landwirtschaft zerfiel nach der Staatsgründung 1993. Tomaten aus Spanien sind billiger als die einheimischen Produkte. Doch zu Essen gibt es mehr als üppig. Die Gastfreundschaft ist gross; wir können über alles offen reden. Bei jedem Empfang sind im Sitzungszimmer belegte Brötchen und Süssigkeiten aufgelegt, nur Broschüren und Prospekte gibt es keine, dafür fehlt das Geld. Aus dem eintönigen Grün leuchten frisch gestrichene Dorfkirchen und auffallend buntgeschmückte Friedhöfe. Entlang der Landstrasse brüten auf hohen Masten die Störche.

Das Gesundheitswesen wird zentralistisch von Bratislava aus organisiert. Weil unstabile Parteikoalitionen regieren, ist meist jede Wahl mit einem Richtungswechsel verbunden. Beklagt werden die fehlende Kontinuität, eine gewisse Konzeptlosigkeit, die extrem kurzen Budgetzeiten und planwirtschaftlich anmutende Vorgaben, die sogar den Einkauf von Lebensmitteln regeln. So muss etwa das Brot in der 400 km entfernten Hauptstadt eingekauft werden. Das Spital in Presov ist ein Zentrum für die Pflegeausbildung und Praktikumsort für Medizinstu- 


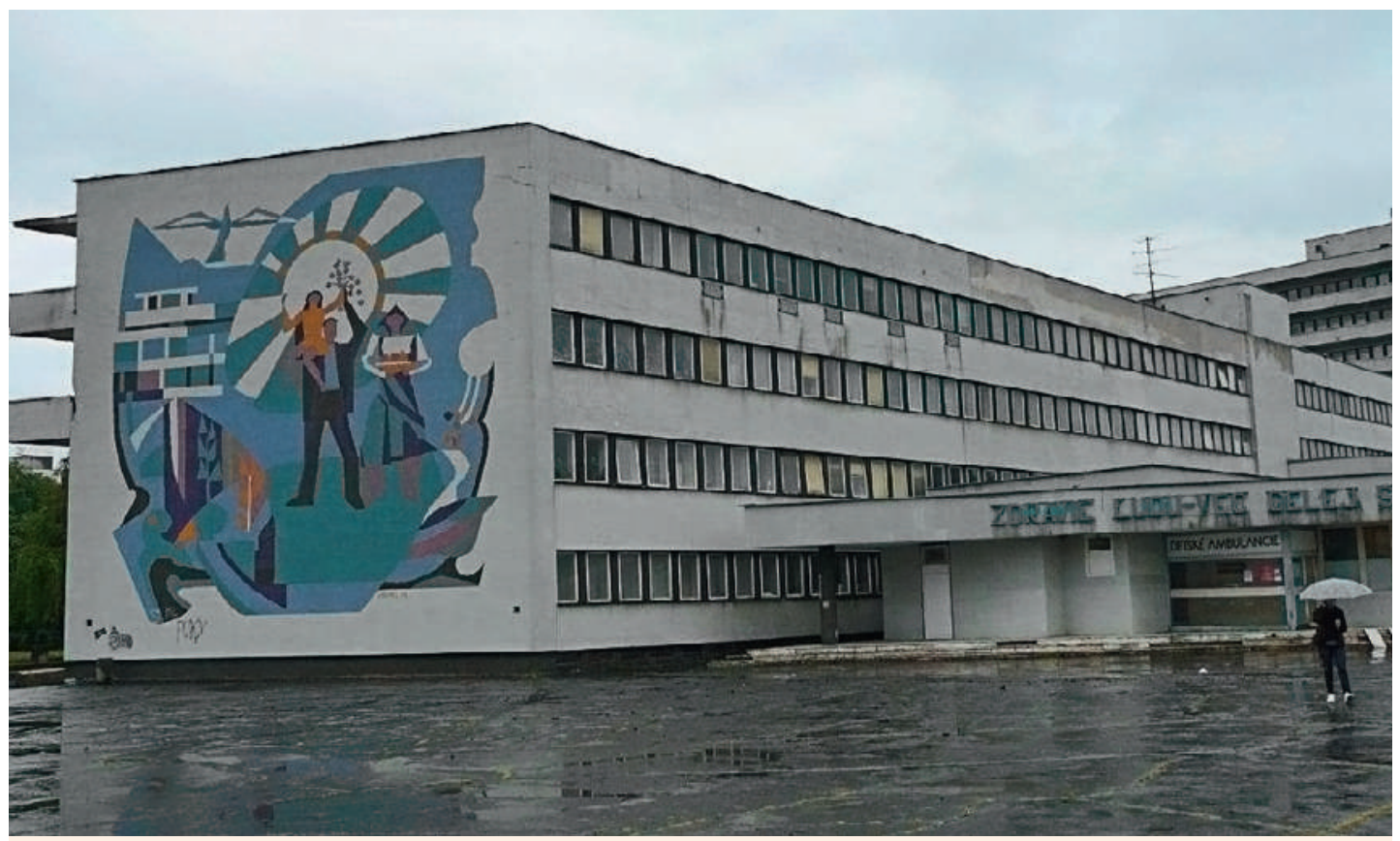

Das Spital in Trebisov.

denten. Vor einem Monat wurde ein sechsstöckiges Gebäude mit EU-Geldern fertiggestellt. In drei Tagen sollen die medizinischen Abteilungen Patienten aufnehmen. Noch werden Schachteln ausgepackt und Kästen eingeräumt. Monitore und Beatmungsgeräte sind angeschlossen, auf dem OP-Tisch im Katheterlabor liegen Instrumente und Ampullen. Für die Matratzen und Bettwäsche auf der Intensivstation hat das Geld nicht gereicht. Wir eilen durch leere Korridore über einen Verbindungsgang in das nebenan liegende Chirurgiegebäude mit Poliklinik und Radiologie im Erdgeschoss. Alt und neu nebeneinander erhöhen den Kontrast. Verglichen mit dem Betrieb eines Kantonsspitals wirkt hier alles still und leer, jede zweite Lampe bleibt aus Spargründen ausser Betrieb.
Stunden. Ein dunkelbrauner, schwarzhaariger Säugling, anonym eingeliefert, wird aufgepäppelt, bis sein Schicksal entschieden ist. Die demographischen Verschiebungen wecken Ängste, denn die Romasippen haben zahlreiche Kinder, mit deren Zulagen sie das Einkommen aufbessern. Sie leben meist abseits in verlotterten Siedlungen und trotz Obligatorium schliessen die wenigsten eine Schule ab. Dabei investiert der Staat viel in die Ausbildung der Jugend.

$\mathrm{Zu}$ den zahlreichen Sendungen der Stiftung «Hilfe und Beistand» des schweizerischen Malteserordens* gehören auch Schulpulte, Hefte, Tafeln und Laptops. Wie das erhaltene Spitalmaterial wird alles, in Zusammenarbeit mit der slowakischen Botschaft, in sehr gutem Zustand gratis geliefert. Freiwillige Mitarbeiter beladen Container und Lastwagen. Zwei

\section{Das Gesundheitswesen wird zentralistisch von Bratislava aus organisiert.}

Das gespendete Material erweitert den Spielraum, denn das eingesparte Geld erlaubt weitere Sanierungen. In Vranov bröckelt die Fassade, doch die Abteilungen für chronisch Kranke und Kinder sind neu gestrichen. Die Gemeinde hat eine Spielecke gesponsert, es gibt Topfpflanzen, Kunstdrucke und fröhlich bemalte Wände. Die Gitterbetten aus der Schweiz sind belegt, denn jährlich kommen hier etwa 900 Kinder zur Welt. Die Hälfte davon Roma. Ein schwieriges Thema, das offiziell keines ist. Während die einheimischen Mütter und Neugeborenen das Spital nach drei bis vier Tagen instruiert verlassen, verschwinden die Romafrauen nach wenigen
Drittel des Zollwertes und die Transportkosten übernimmt das Spital, das dafür die zehn meistbegehrten Gerätschaften erhält. Jede Unterstützung kommt direkt den Patienten und Pflegenden zugute. Ein moderner Ablasshandel für unsere Wegwerfgesellschaft. Ökonomisch gesprochen ist das Land seit 20 Jahren in einer Phase der Transformation. Ein schmerzlicher Übergang mit Gewinnern und Verlierern. Kosice, das zweitgrösste Zentrum des Landes, präsentiert sich als Kulturstadt 2013. Seit 2004 ist die Slowakei ein Mitglied der europäischen Union. Ein kurzer Abstecher in die Ukraine genügt, um uns die Vorteile dieser Gemeinschaft aufzuzeigen. 